Gazi University
Journal of Science
http://dergipark.gov.tr/gujs

\title{
Stochastic Lot Sizing Problem with Carbon Emission Constraints
}

\author{
Esra KOCA* \\ Sabanci University, Industrial Engineering Program, 34956, Tuzla, Istanbul, Turkey.
}

\author{
Highlights \\ - Stochastic lot sizing problem with $\alpha$ service level constraints was studied. \\ - Probabilistic periodic and global carbon emission constraints were considered. \\ - The problems were formulated as mixed integer linear programs. \\ - The effect of probabilistic carbon emission constraints was investigated.
}

\begin{tabular}{l} 
Article Info \\
\hline Received: $30 / 07 / 2019$ \\
Accepted: $15 / 06 / 2020$ \\
Keywords \\
Stochastic lot sizing \\
Green lot sizing \\
Carbon emission \\
Constraints \\
Chance constraints
\end{tabular}

\section{INTRODUCTION}

The climate change and global warming have started to become one of the most important global problems as they negatively affect the social and economic life all over the world. In a recent study by Burke et al. [1], it is stated that if nothing is done, the temperature will increase by 4 degrees Celsius by 2100 , and the global GDP would fall more than 30 percent from 2010 levels which is worse than the Great Depression. Besides, 97 percent of the scientists agree that climate warming is caused by human activities [2]. As a precaution, many countries have started to reduce the greenhouse gases caused by production and distribution processes by imposing legal restrictions. One of the major policies for carbon emission reduction is to limit the carbon emissions of the companies through mandatory emission caps. As a response to this development, recently, carbon emission constraints are started to be included in production and transportation systems.

In this study, we consider a production planning problem with carbon emission constraints. More specifically, we study the stochastic lot sizing problem where the demand follows a stochastic process, and we assume that the firm has a carbon capacity that should be obeyed. We assume that the carbon emission is associated with setting up the production environment, production, and keeping inventory. We consider different versions of the carbon emission constraints and discuss the complexity of the problem, and the effects of the different restrictions on the production strategies and the total carbon emissions.

The lot sizing problem has been studied extensively since the seminal work of Wagner and Whitin [3]. A review of the lot sizing problem and its extensions can be found in the book by Pochet and Wolsey [4]. The deterministic lot sizing problem with carbon emission constraints was studied by several authors under different settings. Benjaafar et al. [5] formulate the lot sizing problems under different carbon emission

$$
\text { *e-mail: ekoca@sabanciuniv.edu }
$$


constraints: carbon capacities, carbon tax, cap-and-trade policy, and carbon offsets. The authors investigate the impact of different emission regulations on the management decisions. Absi et al. [6] study the multi-sourcing uncapacitated lot sizing problem with periodic, cumulative, global and rolling carbon emission constraints. It is assumed that the carbon emission is only caused by production and the average/unitary carbon emission at each period should not exceed an allowed amount. For the periodic constraints a dynamic programming algorithm is developed, and it is showed that the problem is NP-hard under the cumulative and global emission constraints.

Helmrich et al. [7] study the uncapacitated lot sizing problem with global emission constraints where the emissions are due to both production and holding inventories. The authors prove that the problem is NPhard even if the production is the only cause of carbon emissions and the emission amount is the same for each unit produced. Moreover, they show that the problem is still NP-Hard if there exist two production modes, the emissions are linear in the production amounts, and either all cost or emission parameters are the same for all periods. The authors introduce several (non-polynomial) algorithms to find solutions for the problem.

Akbalik and Rapine [8] consider the uncapacitated lot sizing problem with the cap-and-trade carbon policy and the global emission capacity constraint. The authors reduce the problem to the classical uncapacitated lot sizing problem and consequently, show that the problem is polynomially solvable. Nevertheless, the problem becomes NP-hard when there exist budget (capacity) constraints on the carbon emissions.

Romeijn et al. [9] study a bilevel lot sizing problem where the second objective is to minimize the emission costs. The authors make use of both the global and the periodic emission constraints to find the Pareto efficient solutions to the problems, and they discuss the complexity of the problem under different cost structures and emission constraints.

Absi et al. [10] study the uncapacitated lot sizing problem when there exist different production modes with different carbon emissions and production costs. It is assumed that the unitary carbon emission at each period should not be greater than an allowed amount. The emissions are due to the setups and production amounts. The authors show that the problem is NP-hard even if there exist only two production modes and there is no cost for holding inventory.

Hong et al. [11] consider the uncapacitated lot sizing problem with two production modes, a regular and a green technology, under cap-and-trade policy. The authors consider the periodic emission constraints and introduce a dynamic programming algorithm to solve the case where the emission is only due to production.

In all these studies mentioned above, the deterministic version of the lot sizing problem where the demand for the next $\mathrm{T}$ periods is assumed to be known certainly is considered. However, in most of the real-life production systems the demand for the future periods is not known beforehand and estimating the exact demand information may be a challenging task. Thus, the deterministic lot sizing problem may not appropriately represent these systems. In the stochastic lot sizing problem, to represent real life systems better, this assumption is relaxed and it is assumed that the demand is random.

Now we will briefly summarize the related literature on the stochastic lot sizing problem. Silver [12] is one of the first studies on the stochastic lot sizing problem where a heuristic method is proposed for the problem. Laserre et al. [13] study the stochastic lot sizing problem when there exist capacity restrictions for both production and keeping inventory. The authors use chance constraints instead of the stock upper bound constraints and reformulate the problem as a deterministic lot sizing problem. Bookbinder and Tan [14] consider the stochastic lot sizing problem with $\alpha$ service level constraints, $\alpha$ is the lower bound for the probability of satisfying the demand of any period, under the static uncertainty (all the arrangements are done beforehand), dynamic uncertainty (decisions are taken dynamically at each period according to the demand realizations) and static-dynamic uncertainty (replenishment periods are decided at the beginning of the planning horizon, and the amounts that will be produced in these periods are decided dynamically) 
strategies. The dynamic decision-making structure of dynamic and static-dynamic uncertainty rules result in system nervousness even though they might decrease the total system costs $[15,16]$.

Estimating the backlogging or shortage costs might be very hard as they might result in poor customer satisfaction which may not be easily quantified $[5,17]$. This situation makes the service level constraints more popular in the stochastic production planning problems where the backlogging costs are generally omitted when the service levels are high (see Chen and Krass [18] for a review of service level constraints).

To the best of our knowledge, there is only one study by Purohit et al. [19] that is related to ours. Purohit et al. [19] consider the stochastic lot sizing problem with carbon emission constraints under the staticdynamic uncertainty strategy where the production periods are determined beforehand but the actual production amounts are decided at the beginning of the production periods based on the inventory levels observed. As it is stated before, the static-uncertainty strategy is criticized due to the system nervousness it causes by the uncertainty of the production plan. Besides, the authors use the expected values of the production amounts and inventory levels in the carbon emission constraints which may result in large violations in the carbon emission constraints in practice. In this study, we consider the static uncertainty rule and use chance constraint versions of the emission constraints which ensure that the emission constraints will be satisfied by a certain probability level.

There are also several studies on the EOQ type models with carbon emission constraints (e.g. Ghosh et al. [20], Qiao et al. [21] ) where the demand is assumed to be stationary (time-independent). These studies are not related to the lot sizing problems we consider in this paper since the demand is assumed to be dynamic (time-dependent) in our setting.

Our contributions. We employ chance constraints in the context of stochastic lot sizing problem under the static uncertainty rule with carbon emission constraints. To the best of our knowledge, this is the first study on this problem. We develop mixed integer programming formulations that are capable of solving quite large problem instances in reasonable times. We also conduct extensive numerical experiments to demonstrate the value of proposed modeling approach and the effect of different problem parameters on the total costs and the carbon emissions.

This study is important from the practical perspective due to the applicability of the stochastic lot sizing problem in different production, procurement and transportation systems. Besides, including carbon emission constraints in this problem makes the problem more appropriate for today's practice.

The rest of the paper is organized as follows. In section 2, we describe the problem and develop our formulations. In section 3, we present the results of the computational experiments, and we discuss the observations and the future research problems in section 4 .

\section{PROBLEM DEFINITION AND FORMULATIONS}

We consider the stochastic lot sizing problem with service level constraints and carbon emission constraints. We search for the production plan for the next $T$ periods. We assume that the demand of period $t$, represented by $d_{t}$, has normal distribution with mean $\mu_{t}$ and standard deviation $\sigma_{t}$ for $t=1, \ldots, T$. Besides, demands of different periods are independent from each other. Backlogging is allowed but all shortages are satisfied whenever a quantity becomes available. We use $\alpha$ service level constraints to restrict this case, where $\alpha$ is a lower bound for the probability of satisfying the demand of any period. There is no production capacity constraint, but the production will be limited due to the carbon emission constraints. We assume the static uncertainty strategy; thus, all decisions are taken before the demand is realized. Our goal is to determine a feasible production plan that ensures the service level and carbon emission constraints with minimum total expected cost.

Let $x_{t}$ be the production amount in period $t, y_{t}=1$ if there will be a production $\left(x_{t}>0\right)$ in period $t$ and 0 otherwise, and $s_{t}$ be the inventory on hand at the end of period $t$. 
We consider the problem under global and periodic carbon emission constraints. With the global carbon emission constraints, the carbon emitted from production and keeping inventory is limited over the whole planning horizon, whereas in the periodic constraints the carbon emitted at each period is limited separately for each period. Let $\hat{f}_{t}, \hat{p}_{t}, \hat{h}_{t}$ be the carbon emitted from setting up the production environment, production of one unit and keeping a unit in the inventory in period $t$, respectively. If the demand was not random, then the periodic carbon emission constraints would be

$$
\hat{f}_{t} y_{t}+\hat{p}_{t} x_{t}+\hat{h}_{t} s_{t} \leq \hat{C}_{t} \quad t=1, \ldots, T
$$

where the total carbon emission is limited by $\hat{C}_{t}$ for period $t=1, \ldots, T$. Similarly, if the demand was not random, the global carbon emission constraints would have the form

$$
\sum_{t=1}^{T}\left(\hat{t}_{t} y_{t}+\hat{p}_{t} x_{t}+\hat{h}_{t} s_{t}\right) \leq \widehat{K}
$$

where the total carbon emission over the planning horizon is limited by $\widehat{K}$.

When the demand is random, the inventory on hand at the end of each period $s_{t}$ will be a random variable. If there is no carbon emission due to keeping inventory, i.e. $\hat{h}_{t}=0 \forall t$, then constraints (1) and (2) could be used in the formulation directly. Then, the problem is NP-Hard when $\hat{f}_{t}, \hat{p}_{t}$ or $\hat{C}_{t}$ are time-dependent, and it is polynomially solvable when all emission parameters are time-invariant, and the cost parameters are non-speculative [9]. On the other hand, if keeping inventory also causes carbon emission, then we cannot use carbon emission constraints (1) and (2) directly as they include random variables $s$.

In this study, we use chance constraints to ensure that probability of satisfying the carbon emission constraints is greater than or equal to a threshold value. Chance (probabilistic) constraints are one of the well-known approaches to model optimization problems under uncertainty. Chance constrained optimization was first introduced by Charnes and Cooper [22], and since then, it is widely applied to many different optimization problems including production planning, supply chain management, power systems management, and portfolio optimization. The reader is referred to Prekopa [23], Dentcheva [24], and Shapiro et al. [25] for more details on the chance constrained optimization models.

We use disjoint (individual) chance constraints which have the following form

$$
\operatorname{Pr}\{W \mathrm{z} \geq \xi\} \geq \alpha
$$

where $W$ is a deterministic vector, $\mathbf{z}$ is a decision vector, $\xi$ is a random variable, and $\alpha$ is the threshold probability level. We will use the probabilistic versions of constraints (1) and (2) with probability levels $\beta_{t}$ and $\gamma$, respectively. In other words, we will use the following chance constraints instead of constraints (1) and (2), respectively:

$$
\begin{gathered}
\operatorname{Pr}\left\{\hat{f}_{t} y_{t}+\hat{p}_{t} x_{t}+\hat{h}_{t} s_{t} \leq \hat{C}_{t}\right\} \geq \beta_{t} \quad t=1, \ldots, T \\
\operatorname{Pr}\left\{\sum_{t=1}^{T}\left(\hat{f}_{t} y_{t}+\hat{p}_{t} x_{t}+\hat{h}_{t} s_{t}\right) \leq \widehat{K}\right\} \geq \gamma .
\end{gathered}
$$

Let $q_{t}, c^{\prime}$, and $g_{t}$ be the setup, unit production and inventory holding costs for period t, respectively. Our goal is to determine a production plan which ensures the service level constraints and the probabilistic carbon emission constraints with minimum total expected cost. This problem can be formulated as follows. 


$$
\begin{array}{ll}
\min & \sum_{t=1}^{T}\left(q_{t} y_{t}+c^{\prime}{ }_{t} x_{t}+g_{t} \mathbb{E}\left[\max \left(s_{t}, 0\right)\right]\right) \\
\text { s.t. } & s_{t}=\sum_{i=1}^{t} x_{i}-\sum_{i=1}^{t} d_{i} \quad t=1, \ldots, T \\
& \operatorname{Pr}\left\{s_{t} \geq 0\right\} \geq \alpha \\
& \left(1^{\prime}\right) \text { or }\left(2^{\prime}\right) \\
& x_{t} \leq M y_{t} \quad t=1, \ldots, T \\
& x_{t} \geq 0 \quad t=1, \ldots, T \\
& y_{t} \in\{0,1\} \quad t=1, \ldots, T .
\end{array}
$$

In the objective function (3), the total expected cost due to production and keeping inventory is minimized. In constraints (4), the ending inventory for period $t$ is related to the production amounts and the demand. Here, without loss of generality, it is assumed that the initial inventory is zero. Constraints (5) are the $\alpha$ service level constraints that ensure that probability of no stockout is at least $\alpha$ at any period. Constraints (6) relate the production amounts with the setup decisions: if production occurs in period then setup variable should be 1 for period t. The remaining constraints (7) define the types of the decision variables. We will call the problem with the probabilistic periodic carbon emission constraints (1') and the probabilistic global carbon emission constraints (2') as LS-I, and LS-II, respectively.

Since we assume that the demand has normal distribution, and the demand of different periods are independent, as it is done in Koca et al. [26], the $\alpha$ service level constraints (5) can be written as linear constraints

$$
\operatorname{Pr}\left\{s_{t} \geq 0\right\} \geq \alpha \quad t=1, \ldots . T \Rightarrow \sum_{i=1}^{t} x_{i} \geq Z_{\alpha} \sigma_{1 t}+\mu_{1 t} \quad t=1, \ldots, T
$$

where $Z_{\alpha}$ is the $\alpha$-quantile of the standard normal distribution, $\mu_{1 t}=\sum_{i=1}^{t} \mu_{i}$ is the expected total demand until period $\mathrm{t}$, and $\sigma_{1 t}=\sqrt{\sum_{i=1}^{t} \sigma_{i}^{2}}$ is the standard deviation of the total demand until period t. Besides, since $\alpha$ is assumed to be sufficiently large and shortages are satisfied as soon as possible, the expected total inventory cost in the objective function can be estimated as done in Bookbinder and Tan [14]

$$
\sum_{t=1}^{T} g_{t} \mathbb{E}\left[\max \left\{\mathrm{s}_{\mathrm{t}}, 0\right\}\right] \approx \sum_{t=1}^{T} \bar{g}_{t} x_{t}-\sum_{t=1}^{T} g_{t} \mu_{1 t}
$$

where $\bar{g}_{t}=\sum_{i=t}^{T} g_{i}$ for $t=1, \ldots, T$. Then, we can rewrite the objective function (3) as

$$
\sum_{t=1}^{T}\left(q_{t} y_{t}+c_{t} x_{t}\right)
$$

where $c_{t}=c^{\prime}{ }_{t}+\bar{g}_{t}$ for $t=1, \ldots, T$. Note that in the new objective function (10), we ignore the second term in the right-hand side of (9) which is a constant.

Now, we focus on the probabilistic periodic carbon emission constraint (1') for a period t. We will use the notation $x_{1 t}=\sum_{i=1}^{t} x_{i}$ and $d_{1 t}=\sum_{i=1}^{t} d_{i}$ to simplify the expressions. The probabilistic constraints can be expressed in terms of the cumulative probability distribution $G_{d_{1 t}}$ of the total demand until period $\mathrm{t}$ 


$$
\begin{aligned}
\operatorname{Pr}\left\{\hat{f}_{t} y_{t}+\hat{p}_{t} x_{t}+\hat{h}_{t} s_{t} \leq \hat{C}_{t}\right\} & =\operatorname{Pr}\left\{s_{t} \leq \frac{\hat{C}_{t}-\hat{f}_{t} y_{t}-\hat{p}_{t} x_{t}}{\hat{h}_{t}}\right\} \\
& =\operatorname{Pr}\left\{x_{1 t}-d_{1 t} \leq \frac{\hat{C}_{t}-\hat{f}_{t} y_{t}-\hat{p}_{t} x_{t}}{\hat{h}_{t}}\right\} \\
& =\operatorname{Pr}\left\{d_{1 t} \geq \frac{\hat{f}_{t} y_{t}+\hat{p}_{t} x_{t}+\hat{h}_{t} x_{1 t}-\hat{C}_{t}}{\hat{h}_{t}}\right\} \\
& =1-G_{d_{1 t}}\left(\frac{\hat{f}_{t} y_{t}+\hat{p}_{t} x_{t}+\hat{h}_{t} x_{1 t}-\hat{C}_{t}}{\hat{h}_{t}}\right)
\end{aligned}
$$

and consequently, constraint ( $\left.1^{\prime}\right)$ can be rewritten as a linear inequality:

$$
\begin{gathered}
\operatorname{Pr}\left\{\hat{f}_{t} y_{t}+\hat{p}_{t} x_{t}+\hat{h}_{t} s_{t} \leq \hat{C}_{t}\right\} \geq \beta_{t} \Rightarrow \frac{\hat{f}_{t}}{\hat{h}_{t}} y_{t}+\frac{\hat{p}_{t}}{\hat{h}_{t}} x_{t}+x_{1 t} \leq \frac{\hat{C}_{t}}{\hat{h}_{t}}+\mu_{1 t}+Z_{1-\beta_{t}} \sigma_{1 t} \\
\Rightarrow \bar{f}_{t} y_{t}+\bar{p}_{t} x_{t}+\sum_{i=1}^{t} x_{i} \leq \bar{C}_{t}
\end{gathered}
$$

where $\bar{f}_{t}=\frac{\hat{f}_{t}}{\hat{h}_{t}}, \bar{p}_{t}=\frac{\hat{p}_{t}}{\widehat{h}_{t}}$ and $\bar{C}_{t}=\frac{\hat{C}_{t}}{\hat{h}_{t}}+\mu_{1 t}+Z_{1-\beta_{t}} \sigma_{1 t}$. As a result, we reformulate LS-I as a mixed integer linear program:

$$
\begin{aligned}
& (L S-P) \\
& \min \sum_{t=1}^{T}\left(q_{t} y_{t}+c_{t} x_{t}\right) \\
& \text { s.t. } \sum_{i=1}^{t} x_{i} \geq Z_{\alpha} \sigma_{1 t}+\mu_{1 t} \quad t=1, \ldots, T \\
& \bar{f}_{t} y_{t}+\bar{p}_{t} x_{t}+\sum_{i=1}^{t} x_{i} \leq \bar{C}_{t} \quad t=1, \ldots, T \\
& \text { (6) - (7) }
\end{aligned}
$$

Note that constraints (8) and (11) are similar to the demand satisfaction and production capacity constraints of the classical lot sizing problem. But here the capacity constraints also include the previous production amounts. Indeed, constraints (11) are very similar to their deterministic counterparts (1) where the total demand until t, $d_{1 t}$ is replaced by $Z_{1-\beta_{t}} \sigma_{1 t}+\mu_{1 t}$. Due to Romeijn et al. [9], LS-P is polynomially solvable when all emission parameters are time-independent, and it is NP-Hard when $\bar{f}_{t}$ or $\bar{p}_{t}$ are time-variant. In the next section, we will present the computational results for this formulation.

Now, we will rewrite the probabilistic global carbon emission constraint (2') as a linear constraint. The probabilistic constraint can be expressed as 


$$
\begin{aligned}
\operatorname{Pr}\left\{\sum_{t=1}^{T}\left(\hat{f}_{t} y_{t}+\hat{p}_{t} x_{t}+\hat{h}_{t} s_{t}\right) \leq \widehat{K}\right\} & =\operatorname{Pr}\left\{\sum_{t=1}^{T} \hat{h}_{t} s_{t} \leq \widehat{K}-\sum_{t=1}^{T} \hat{f}_{t} y_{t}-\sum_{t=1}^{T} \hat{p}_{t} x_{t}\right\} \\
& =\operatorname{Pr}\left\{\sum_{t=1}^{T} \hat{h}_{t}\left(x_{1 t}-d_{1 t}\right) \leq \widehat{K}-\sum_{t=1}^{T} \hat{f}_{t} y_{t}-\sum_{t=1}^{T} \hat{p}_{t} x_{t}\right\} \\
& =\operatorname{Pr}\left\{\sum_{t=1}^{T} \hat{h}_{t} d_{1 t} \geq \sum_{t=1}^{T} \hat{f}_{t} y_{t}+\sum_{t=1}^{T} \hat{p}_{t} x_{t}+\sum_{t=1}^{T} \hat{h}_{t} x_{1 t}-\widehat{K}\right\} \\
& =1-\operatorname{Pr}\left\{\sum_{t=1}^{T} \tilde{h}_{t} d_{t} \leq \sum_{t=1}^{T} \hat{f}_{t} y_{t}+\sum_{t=1}^{T} \tilde{p}_{t} x_{t}-\widehat{K}\right\}
\end{aligned}
$$

where $\tilde{h}_{t}=\sum_{i=t}^{T} \hat{h}_{i}$ and $\tilde{p}_{t}=\hat{p}_{t}+\sum_{i=t}^{T} \hat{h}_{i}$. As $d_{t}$ is normally distributed with mean $\mu_{t}$ and standard deviation $\sigma_{t}$, and as they are independent for each period, $\sum_{t=1}^{T} \tilde{h}_{t} d_{t}$ has also normal distribution with mean $\tilde{\mu}=\sum_{t=1}^{T} \tilde{h}_{t} \mu_{t}$ and standard deviation $\tilde{\sigma}=\sqrt{\sum_{t=1}^{T} \tilde{h}_{t}^{2} \sigma_{t}^{2}}$. Thus, constraint (2') can be rewritten as a linear inequality

$$
\operatorname{Pr}\left\{\sum_{t=1}^{T}\left(\hat{f}_{t} y_{t}+\hat{p}_{t} x_{t}+\hat{h}_{t} s_{t}\right) \leq \widehat{K}\right\} \geq \gamma \Rightarrow \sum_{t=1}^{T} \hat{f}_{t} y_{t}+\sum_{t=1}^{T} \tilde{p}_{t} x_{t} \leq \widehat{K}+Z_{1-\gamma} \tilde{\sigma}+\tilde{\mu} .
$$

Consequently, LS-II can be reformulated as a mixed integer program by replacing (11) with (12) in the formulation LS-P, which will be called as LS-G. Note that LS-G is also NP-Hard even if only $\tilde{p}_{t}$ is positive due to Helmrich et al. [7].

The mixed integer linear programs LS-P and LS-G are obtained under the normally distributed demand assumption. If this is not the case, a closed form formulation for the problems may not be obtained easily; but, again, the problems can be formulated as mixed integer programs given that all $G_{d_{1 t}}^{-1}($.$) values that will$ be used in the models are calculated beforehand.

In the next section, we will present the computational experiments for the formulations LS-P and LS-G and discuss their effects on the total costs and the carbon emissions.

\section{COMPUTATIONAL EXPERIMENTS}

We test the formulations LS-P and LS-G in terms of their capabilities to solve large problem instances and investigate the effect of probabilistic carbon emission constraints in the total expected cost and the carbon emissions.

We generate test instances as follows. We consider the problem instances for $T=50,100$ periods. We assume that the unit inventory holding cost $(g)$ is 1 and unit production cost (c) is 5 for all periods. Different time-invariant setup costs $q \in\{1000,5000\}$ are considered. We assume that coefficient of variation is $10 \%$, and the expected demand for each period is determined as $\mu_{t} \sim U[0.7 \mu, 1.3 \mu]$ for $t=1, \ldots, T$ where $\mu \in\{100,1000\}$. The carbon emission parameters are assumed to be time-invariant and selected as follows: $\hat{h}=1, \quad \hat{p}=3, \quad \hat{f}=0.1 q, \quad \hat{C}=a C_{\max } \quad$ for $\quad a \in\{0.03,0.05,0.08\}$ when $T=50, \quad$ and $\quad a \in$ $\{0.02,0.03,0.05,0.08\}$ when $T=100$ where $C_{\max }$ is the carbon capacity that allows to produce the amount $\mu_{1 T}+Z_{\alpha} \sigma_{1 T}$ and keep it in inventory. To be able to compare the models with periodic and global emission constraints, we select the global carbon emission capacities as $\widehat{K}=k T \hat{C}$ where $k \in\{0.5,0.8,1\}$ and $k \in\{0.2,0.3,0.5,0.8\}$ for 50 and 100 period problem instances, respectively. We assume that $\alpha=0.90$ and consider different probability levels for emission constraints: $\beta_{t}=\beta$ for all $t$, and $\beta=\gamma \in$ $\{0.90,0.95,0.98\}$.

We implement the formulations in Gurobi 8.0.1 and perform the experiments on a $2.60 \mathrm{GHz}$ Intel Core i7 machine with 16GB memory running Windows 10 . We set the time limit to 3600 seconds. 
We first discuss the solution times of LS-P. For each instance group, we report the average solution times (for the instances solved to optimality), the percentage of instances that cannot be solved within the time limit, and the average final gaps for those instances in Table 1 . Note that all 50 period problem instances are solved in very short computation times, the average and maximum solution times for these instances are 8 and 147 seconds, respectively. Similarly, 100 period instances are also solved in short times when $\mu=$ 1000, the maximum solution times are 2 and 429 seconds for $q=1000$ and $q=5000$, respectively. On the other hand, we could not solve $33 \%$ of the instances when $\mu=100$. We observe that it is harder to solve the problem when the setup costs are higher, and the emission capacities are tighter. Similarly, the problem becomes difficult when $\beta$ is larger (not reported in Table 1), which makes the modified emission capacity $\bar{C}$ smaller. As it can be seen from Table 1 , we could not solve none of the instances when set up cost is 5000, and the emission capacity coefficient is 0.02 and 0.03 . But note that the reported average final gaps are very small.

We solve the same problem instances with different $a$ and $\gamma$ values by LS-G and summarize the results in Table 2. We ignore some of the parameter settings that cause infeasibility due to smaller emission capacities, such as $a=0.03, k=0.5$ and $\gamma=0.95$. We again solve all instances with $\mathrm{T}=50$ optimally within the time limit by LS-G. For 50 period instances, the maximum solution time is 64 seconds, and average is 2.43 seconds. When $T=100$, instances with $\mu=1000$ are solved approximately in 5 seconds, the maximum solution time is 13 seconds for this class. As it can be seen from Table 2, it is harder to solve the problem when setup costs are higher and the emission capacities (when $a \times k$ ) are smaller. Note that we observe the same result for LS-P. But, instances with $q=5000$ are solved in very short times compared to LS-P. Only in 57\% of the instances with $q=5000, a \times k \in\{0.015,0.016\}$ LS-G could not be solved optimally, the average final gap for these instances is $1.26 \%$.

Table 1. Average solution times and final gaps of LS-P

\begin{tabular}{|c|c|c|c|c|c|c|}
\hline$T$ & $\mu$ & $q$ & $a$ & Time (sec) & $\%$ not solved & fgap (\%) \\
\hline \multirow[t]{12}{*}{50} & \multirow[t]{6}{*}{100} & \multirow[t]{3}{*}{1000} & 0.03 & 6 & $0 \%$ & $\mathrm{NA}$ \\
\hline & & & 0.05 & 5 & $0 \%$ & $\mathrm{NA}$ \\
\hline & & & 0.08 & 2 & $0 \%$ & NA \\
\hline & & \multirow[t]{3}{*}{5000} & 0.03 & 1 & $0 \%$ & NA \\
\hline & & & 0.05 & 1 & $0 \%$ & NA \\
\hline & & & 0.08 & 1 & $0 \%$ & NA \\
\hline & \multirow[t]{6}{*}{1000} & \multirow[t]{3}{*}{1000} & 0.03 & 26 & $0 \%$ & $\mathrm{NA}$ \\
\hline & & & 0.05 & 100 & $0 \%$ & NA \\
\hline & & & 0.08 & 11 & $0 \%$ & $\mathrm{NA}$ \\
\hline & & \multirow[t]{3}{*}{5000} & 0.03 & 3 & $0 \%$ & NA \\
\hline & & & 0.05 & 3 & $0 \%$ & $\mathrm{NA}$ \\
\hline & & & 0.08 & 1 & $0 \%$ & $\mathrm{NA}$ \\
\hline \multirow[t]{8}{*}{100} & \multirow[t]{8}{*}{100} & \multirow[t]{4}{*}{1000} & 0.02 & 1405 & $33 \%$ & 0.07 \\
\hline & & & 0.03 & 295 & $33 \%$ & 0.02 \\
\hline & & & 0.05 & 11 & $0 \%$ & $\mathrm{NA}$ \\
\hline & & & 0.08 & 10 & $0 \%$ & NA \\
\hline & & \multirow[t]{4}{*}{5000} & 0.02 & $\mathrm{NA}$ & $100 \%$ & 0.58 \\
\hline & & & 0.03 & NA & $100 \%$ & 0.52 \\
\hline & & & 0.05 & 291 & $0 \%$ & NA \\
\hline & & & 0.08 & 47 & $0 \%$ & $\mathrm{NA}$ \\
\hline
\end{tabular}




\begin{tabular}{|c|c|c|c|c|c|}
\hline \multirow[t]{8}{*}{1000} & \multirow[t]{4}{*}{1000} & 0.02 & 1 & $0 \%$ & $\mathrm{NA}$ \\
\hline & & 0.03 & 1 & $0 \%$ & NA \\
\hline & & 0.05 & 1 & $0 \%$ & NA \\
\hline & & 0.08 & 1 & $0 \%$ & NA \\
\hline & \multirow[t]{4}{*}{5000} & 0.02 & 340 & $0 \%$ & NA \\
\hline & & 0.03 & 26 & $0 \%$ & NA \\
\hline & & 0.05 & 5 & $0 \%$ & NA \\
\hline & & 0.08 & 4 & $0 \%$ & NA \\
\hline
\end{tabular}

Table 2. Average solution times and final gaps of $L S-\mathrm{G}$

\begin{tabular}{|c|c|c|c|c|c|c|}
\hline$T$ & $\mu$ & $q$ & $a \times k$ & Time (sec) & $\%$ not solved & fgap (\%) \\
\hline \multirow[t]{12}{*}{50} & \multirow[t]{6}{*}{100} & \multirow[t]{3}{*}{1000} & $\{0.024,0.025\}$ & 1 & $0 \%$ & NA \\
\hline & & & $\{0.03,0.04,0.05\}$ & 1 & $0 \%$ & NA \\
\hline & & & $\{0.064,0.08\}$ & 2 & $0 \%$ & NA \\
\hline & & \multirow{3}{*}{5000} & $\{0.024,0.025\}$ & 16 & $0 \%$ & NA \\
\hline & & & $\{0.03,0.04,0.05\}$ & 2 & $0 \%$ & NA \\
\hline & & & $\{0.064,0.08\}$ & 2 & $0 \%$ & NA \\
\hline & \multirow[t]{6}{*}{1000} & \multirow[t]{3}{*}{1000} & $\{0.024,0.025\}$ & 1 & $0 \%$ & NA \\
\hline & & & $\{0.03,0.04,0.05\}$ & 1 & $0 \%$ & NA \\
\hline & & & $\{0.064,0.08\}$ & 1 & $0 \%$ & NA \\
\hline & & \multirow[t]{3}{*}{5000} & $\{0.024,0.025\}$ & 1 & $0 \%$ & NA \\
\hline & & & $\{0.03,0.04,0.05\}$ & 1 & $0 \%$ & NA \\
\hline & & & $\{0.064,0.08\}$ & 1 & $0 \%$ & NA \\
\hline \multirow[t]{12}{*}{100} & \multirow[t]{6}{*}{100} & \multirow[t]{3}{*}{1000} & $\{0.015,0.016\}$ & 25 & $0 \%$ & NA \\
\hline & & & $\{0.024,0.025\}$ & 16 & $0 \%$ & NA \\
\hline & & & $\{0.04,0.064\}$ & 17 & $0 \%$ & NA \\
\hline & & \multirow[t]{3}{*}{5000} & $\{0.015,0.016\}$ & 180 & $57 \%$ & 1.26 \\
\hline & & & $\{0.024,0.025\}$ & 240 & $0 \%$ & NA \\
\hline & & & $\{0.04,0.064\}$ & 483 & $0 \%$ & NA \\
\hline & \multirow[t]{6}{*}{1000} & \multirow[t]{3}{*}{1000} & $\{0.015,0.016\}$ & 1 & $0 \%$ & NA \\
\hline & & & $\{0.024,0.025\}$ & 1 & $0 \%$ & $\mathrm{NA}$ \\
\hline & & & $\{0.04,0.064\}$ & 1 & $0 \%$ & NA \\
\hline & & \multirow[t]{3}{*}{5000} & $\{0.015,0.016\}$ & 7 & $0 \%$ & NA \\
\hline & & & $\{0.024,0.025\}$ & 8 & $0 \%$ & NA \\
\hline & & & $\{0.04,0.064\}$ & 10 & $0 \%$ & NA \\
\hline
\end{tabular}

According to the results given in Tables 1 and 2, we conclude that it is harder to solve LS-P, in general. Note that when $k=1$, LS-P and LS-G have the same total emission capacities, but as LS-P has periodic capacity constraints it will be more restricted than LS-G. We do not consider the case $k=1$ when $\mathrm{T}=100$ 
since even with $k=0.8$, the problem is solved in very short times (when $a=0.05$ and $a=0.08$ ), and the emission constraints become redundant.

Now we will analyze the effect of carbon emission constraints in the total costs by comparing the objective function values of LS-P (and LS-G) and the formulation of classical lot sizing problem with no carbon emission constraints which can be obtained by removing (11) from LS-P (called LS' from now on). We compute the percentage increase in the total cost as $\frac{z^{L S-P}-z^{L S \prime}}{z^{L S \prime}} * 100$ where $z^{L S-P}$ and $z^{L S^{\prime}}$ represent the best upper bounds for LS-P and LS', respectively. Here, if the problem instance is solved to optimality within the time limit, then the best upper bound is equal to the optimal value; otherwise it is the best objective function value obtained by the solver within the time limit. In Figure 1, the data is grouped as $q-\mu-a$ and the average percentage increase in the total cost for each instance group is presented for 50 and 100 period instances, separately. Since there is no change in the total cost when the modified carbon emission capacities $\bar{C}$ are larger (i.e. $a$ and/or $\beta$ are larger) or $\mu=1000$, we do not present the results for those instances in the figure. The numbers above the bars represent the ratio of number of production periods in the solutions of LS-P and LS'.

As it can be observed from Figure 1, the total cost is more affected when the setup costs are higher and the emission capacity coefficient $a$ is smaller. Note that for these instances the ratio of the production periods is very large, 8 and 6.2 for 50 and 100 periods, respectively. For example, if the demand for the next 50 periods is satisfied by producing in 5 periods without carbon emission constraints, now there will be around 40 production periods. The main reason of this situation is the smaller emission capacities for each period. When the modified emission capacities $\bar{C}$ are smaller, less amount could be produced in the production periods, and therefore the total number of production periods, and consequently the total cost, increases. As it is expected, the increase in the total cost is higher when the setup costs are higher.
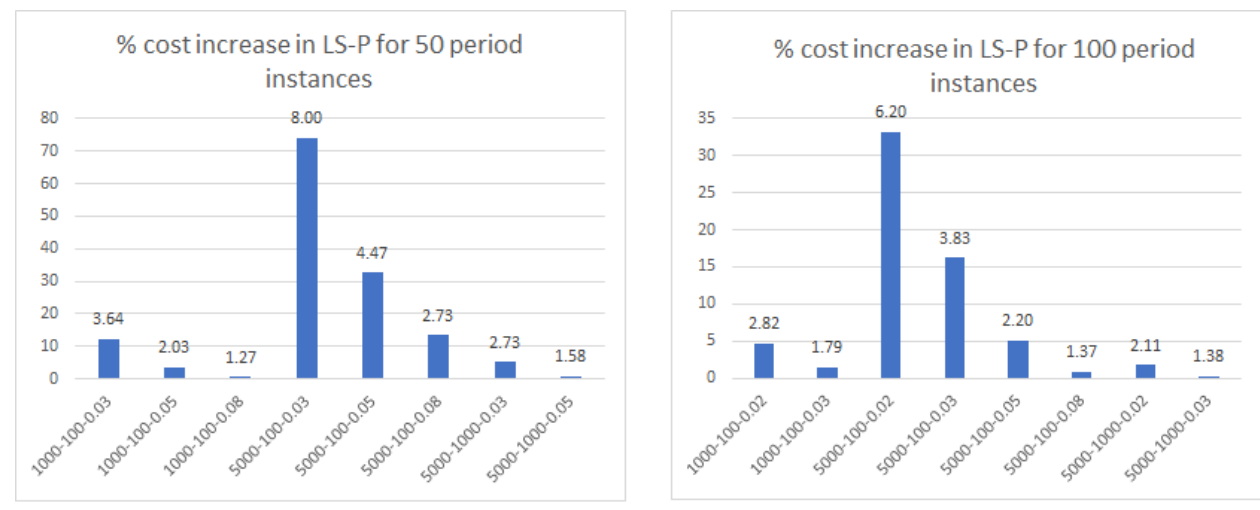

Figure 1. Cost change due to the periodic carbon emission constraints

We make a similar analysis for LS-G. We see that the same solution with LS' is obtained by LS-G when $a \times k \geq 0.04$ and $\mathrm{T}=50$, or $a \times k \geq 0.024$ and $\mathrm{T}=100$, which means that the global emission capacity is sufficient in these settings. Besides, even when the emission capacities are not sufficiently large to use the solution of LS', smaller increases in the total cost are observed. When T $=50$ and $a \times k \in$ $\{0.024,0.025\}$, the maximum cost increase is $5.88 \%$ which is observed when $q=5000$ and $\mu=100$. Only in $18 \%$ of the instances the cost change is positive, and the average cost increase is $1.63 \%$ for these instances. We observe similar results for the instances with $\mathrm{T}=100$ and $a \times k \in\{0.015,0.016\}$, the maximum and average cost increases are $4.35 \%$ and $0.81 \%$, respectively, which is seen only in $11 \%$ of the instances.

The results presented above imply that LS-G is relatively easier to solve, and it results in less cost increase compared to LS-P. Now we will compare the carbon emissions of the production plans obtained by LS-P and LS-G. As the demand for each period $d_{t}$, and consequently the inventory level at the end of each period $s_{t}$ are random, we use simulation to compare the carbon emissions. We select an instance with $T=50, q=$ $5000, \mu=100, \beta=0.1, a=0.05$ for which the emission capacity is $\hat{C}=1535.67$. We generate the 
demand of each period with the specified mean $\mu_{t}$ and the standard deviation $\sigma_{t}$, and determine the inventory level at the end of each period $s_{t}$ by implementing the production plans obtained by LS-P or LSG. We repeat this process for 100 times and summarize the results in Table 3. As LS-G restricts the carbon emission over the whole planning horizon, the first row (\% of periods with violated emission constraints) is not applicable (NA) for it. We observe that there exists approximately one period (out of 50 periods) with violated emission constraint when the solution of LS-P is implemented. As it can be seen from Table 2 , violation of periodic emission constraints occurs in 28 instances out of 100 whereas the global emission constraints are violated in only 5 instances when $\mathrm{k}=0.5$, i.e. the total carbon emission capacity of LS-G is as half as the total emission capacity of LS-P. Besides, when $\mathrm{k}=0.8$ no emission constraint is violated in the solutions of LS-G, and the same solutions are obtained by LS-G and LS'. The most important result of this experiment can be seen in the last row of Table 2: even though the total carbon emission capacity of LS-G is half of the total carbon emission capacity of LS-P, we observe less total carbon emission in LS-P. The main reason of this situation is the differences between the number of periods of the solutions: in the solution of LS-P there exist a production period in every 2 or 3 periods, whereas in the solution of LS-G we see a production period in every 5-6 periods. In other words, in LS-G more is produced and kept in the inventory compared to LS-P. The emission due to holding inventory is $22 \%$ and $43 \%$ of the total emission occurred in LS-P and LS-G, respectively.

Table 3. Comparison of carbon emissions of $L S-P$ and $L S-G$

\begin{tabular}{|c|c|c|c|}
\hline \multirow{2}{*}{} & \multirow{2}{*}{ LS-P } & \multicolumn{2}{|c|}{ LS-G } \\
\cline { 3 - 4 } & & $\mathrm{k}=0.5$ & $\mathrm{k}=0.8$ \\
\hline \% of periods with violated emission constraints & 1.82 & $\mathrm{NA}$ & $\mathrm{NA}$ \\
\hline \% of instances with violated emission constraints & 28 & 5 & 0 \\
\hline Average total carbon emission (for 50 periods) & 33,313 & 34,446 & 43,660 \\
\hline
\end{tabular}

\section{CONCLUSIONS}

We study the stochastic lot sizing problem with the probabilistic periodic and global carbon emission constraints which is not studied in the literature before. We formulate the problem as MIP under the assumption that the demand follows normal distribution and solve the formulations by a commercial solver. In the computational experiments, we see that both formulations LS-P and LS-G can be solved for quite large problem instances in short computation times. These formulations might be tightened, or stronger reformulations can be considered (similar to the ones developed for classical LS) in a future research.

Since LS-P is more restrictive it results in more cost increase compared to the case with no emission constraints. On the other hand, according to our results the average total carbon emissions of the solutions are very close when the total emission capacity of LS-G is as half of the total capacity of LS-P.

Here we consider the problem under the static uncertainty strategy and the $\alpha$ service level constraints. It would be interesting to study the similar problems with the other uncertainty strategies, or joint chance constraints.

\section{CONFLICTS OF INTEREST}

No conflict of interest was declared by the author.

\section{REFERENCES}

[1] Burke, M., Davis, W.M., Diffenbaugh, N.S., "Large potential reduction in economic damages under UN mitigation targets", Nature, 557, 7706: 549, (2018).

[2] Internet: Global Climate Change, https://climate.nasa.gov.tr (July, 2019). 
[3] Wagner, H.M., Whitin, T.M., "Dynamic version of the economic lot size model", Management Science, 5, 1: 89-96, (1958).

[4] Pochet, Y., Wolsey, L.A., "Production planning by mixed integer programming", Springer Science \& Business Media, (2006).

[5] Benjafaar, S., Li, Y., Daskin, M., "Carbon footprint and the management of supply chains: Insights from simple models", IEEE Transactions on Automation Science and Engineering, 10, 1: 99-116, (2012).

[6] Absi, N., Dauzère-Pérès, S., Kedad-Sidhoum, S., Penz, B., Rapine, C., "Lot sizing with carbon emission constraints", European Journal of Operational Research, 227, 1: 55-61, (2013).

[7] Helmrich, M.J.R., Jans, R., Van den Heuvel, W., Wagelmans, A.P., "The economic lot-sizing problem with an emission capacity constraint", European Journal of Operational Research, 241, 1: 50-62, (2015).

[8] Akbalik, A., Rapine, C., "Single-item lot sizing problem with carbon emission under the cap-and-trade policy", 2014 International Conference on Control, Decision and Information Technologies (CoDIT), IEEE, (2014).

[9] Romeijn, H.E., Morales, D.R., Van den Heuvel, W., "Computational complexity of finding Pareto efficient outcomes for biobjective lot-sizing models", Naval Research Logistics (NRL), 61, 5: 386-402, (2014).

[10] Absi, N., Dauzère-Pérès, S., Kedad-Sidhoum, S., Penz, B., Rapine, C., "The single-item green lotsizing problem with fixed carbon emissions", European Journal of Operational Research, 248, 3: 849$855,(2016)$.

[11] Hong, Z., Chu, C., Yu, Y., "Dual-mode production planning for manufacturing with emission constraints", European Journal of Operational Research, 251, 1: 96-106, (2016).

[12] Silver, E., "Inventory control under a probabilistic time-varying, demand pattern", AIIE Transactions, 10, 4: 371-379, (1978).

[13] Lasserre, J.B., Bes, C., Roubellat, F., "The stochastic discrete dynamic lot size problem: an open-loop solution", Operations Research, 33, 3: 684-689, (1985).

[14] Bookbinder, J.H., Tan, J.-Y., "Strategies for the probabilistic lot-sizing problem with service-level constraints", Management Science, 34, 9: 1096-1108, (1988).

[15] Tunc, H., Kilic, O.A., Tarim, S.A., Eksioglu, B., "A simple approach for assessing the cost of system nervousness", International Journal of Production Economics, 141, 2: 619-625, (2013).

[16] Tunc, H., Kilic, O.A., Tarim, S.A., Eksioglu, B., "A reformulation for the stochastic lot sizing problem with service-level constraints", Operations Research Letters, 42, 2: 161-165, (2014).

[17] Nahmias, S., Cheng, Y., "Production and operations analysis", Vol. 6. New York: McGraw-Hill, (2005).

[18] Chen, F.Y., Krass, D., "Inventory models with minimal service level constraints", European Journal of Operational Research, 134, 1: 120-140, (2001).

[19] Purohit, A. K., Choudhary, D., Shankar R., "Inventory lot-sizing under dynamic stochastic demand with carbon emission constraints", Procedia-Social and Behavioral Sciences, 189: 193-197, (2015). 
[20] Ghosh, A., Jha, J.K., Sarmah, S.P., "Optimal lot-sizing under strict carbon cap policy considering stochastic demand", Applied Mathematical Modelling, 44: 688-704, (2017).

[21] Qiao, A., Choi, S.H., Wang, X.J., Zhao, Y.C., "Stochastic lot-sizing under carbon emission control for profit optimisation in MTO manufacturing", In MATEC Web of Conferences, 95: 18003, EDP Sciences, (2017).

[22] Charnes, A., Cooper, W.W., "Deterministic equivalents for optimizing and satisficing under chance constraints", Operations Research, 11: 18-39, (1963).

[23] Prekopa, A., "Handbooks in operations research and management science", Vol 10, Chapter Probabilistic Programming, Elsevier, Amsterdam, Editors: A. Ruszczynski and A. Shapiro, (2003).

[24] Dentcheva, D., "Probabilistic and randomized methods for design under uncertainty", Chapter Optimization Models with Probabilistic Constraints, Springer-Verlag, London, Editors: G. Calafiore and F. Dabbene, (2006).

[25] Shapiro, A., Dentcheva, D., Ruszczynski, A., "Lectures on stochastic programming: modeling and theory", The Society for Industrial and Applied Mathematics and The Mathematical Programming Society, Philadelphia, USA, (2009).

[26] Koca, E, Yaman, H., Aktürk, M.S., "Stochastic lot sizing problem with controllable processing times", Omega, 53: 1-10, (2015). 